\title{
ECG Denoising by Modeling Wavelet Sub-Band Coefficients using Kernel Density Estimation
}

\author{
Shubhada Ardhapurkar*, Ramchandra Manthalkar** and Suhas Gajre ${ }^{* \star}$
}

\begin{abstract}
Discrete wavelet transforms are extensively preferred in biomedical signal processing for denoising, feature extraction, and compression. This paper presents a new denoising method based on the modeling of discrete wavelet coefficients of ECG in selected sub-bands with Kernel density estimation. The modeling provides a statistical distribution of information and noise. A Gaussian kernel with bounded support is used for modeling sub-band coefficients and thresholds and is estimated by placing a sliding window on a normalized cumulative density function. We evaluated this approach on offline noisy ECG records from the Cardiovascular Research Centre of the University of Glasgow and on records from the MIT-BIH Arrythmia database. Results show that our proposed technique has a more reliable physical basis and provides improvement in the Signal-to-Noise Ratio (SNR) and Percentage RMS Difference (PRD). The morphological information of ECG signals is found to be unaffected after employing denoising. This is quantified by calculating the mean square error between the feature vectors of original and denoised signal. MSE values are less than 0.05 for most of the cases.
\end{abstract}

Keywords-Kernel Density Estimation, Discrete Wavelet Transform, Probability Density Function (PDF), Signal to Noise Ratio

\section{INTRODUCTION}

The Electrocardiogram (ECG) reflects the performance and the properties of the human heart and conveys very important information about heart physiology. During recording, ECGs are inevitably contaminated by different kinds of noises and artifacts. The noise is basically interference with the ECG signal and artifacts are transient interruptions. The ECG signal contaminations are due to two main reasons. The first reason is the physical parameters of the recording instrument (electrode artifacts, line noise) and the second reason is the bioelectric activity of the cells not belonging to the area of diagnostic interest (muscle noise, respiration). Unfortunately these contaminations can be present within the spectral support of the ECG and may disturb the clinically important information of signal. Therefore denoising is essential prior to recognition of ECG wave features.

Different techniques have been introduced in the last few decades for ECG denoising. Some contributions have proposed the use of linear or nonlinear filters [1]. However the filtering pro-

\footnotetext{
Manuscript received June 6, 2012; first revision July 6, 2012; accepted Octocber 5, 2011.

Corresponding Author: Shubhada Ardhapurkar

* Dept of Electronics and Telecommunication, ICEEM, Waluj, Aurangabad, Maharashtra, INDIA (vaidya_shubha21 (a)yahoo.com)

** Dept of Electronics and Telecommunication, SGGS Institute of Engineering and Technology, Nanded, Maharashtra, INDIA (rmanthalkar@yahoo.com, suhasgajre@gmail.com)
} 
cess may cause a removal of significant frequencies contributing to ECG features and therefore ECG signals become distorted.

At present, wavelet based denoising methods are well accepted, as these algorithms are superior to nonlinear and linear filtering [2, 3]. Donoho and Johnstone proposed a universal threshold for the wavelet domain, which gives good results [4]. Mikhled Alfouri and Khaled Daqrouq have presented a wavelet transform based approach by designing level dependent thresholds [5]. The method offers low SNR and more diagnostic distortion. Many methods have focused on the reduction of noise due to power line interference, electrode contact noise, muscle contraction noise (electromyographic, EMG), or baseline drift [6-8]. Denoising methods based on adaptive filters for ECG analysis are widely accepted. However, such algorithms are unable to track the rapidly varying ECG signal $[9,10]$. L. N. Sharma et al. have proposed a denoising method based on an evaluation of higher-order statistics like kurtosis and energy contribution efficiency [11]. Muhammad Zia Ur Rahman et al. have developed efficient sign based normalised adaptive filtering for the cancellation of artifacts in an ECG [12].

In this paper, statistical distribution of the wavelet coefficients of ECG signals are examined by modeling. Using the Gaussian kernel density function at selected levels does this. The appropriate thresholds are designed over a cumulative density function. This approach basically eliminates the interfering of high-density smaller coefficients with low-density significant coefficients.

The rest of this paper is organized as follows. Section II, describes the basics of discrete wavelet based denoising and Gaussian kernel density estimation. The practical implementation supporting the theoretical basis of our approach on the noisy ECG signal is also explained in this section. Section III gives the results of our experimentation, illustrating the comparison and evaluation of diagnostic distortion.

\section{RELATED WORK}

\subsection{Wavelet Based Denoising}

In wavelet based denoising methods, noise corrupted ECG signals are decomposed up to the proper level. The ECG and noises have different variation characters. The values of wavelet coefficients describe the variation on the time frequency scale. Thresholds are designed by using standard deviation or a variance of coefficients. Then either hard or soft thresholding is applied

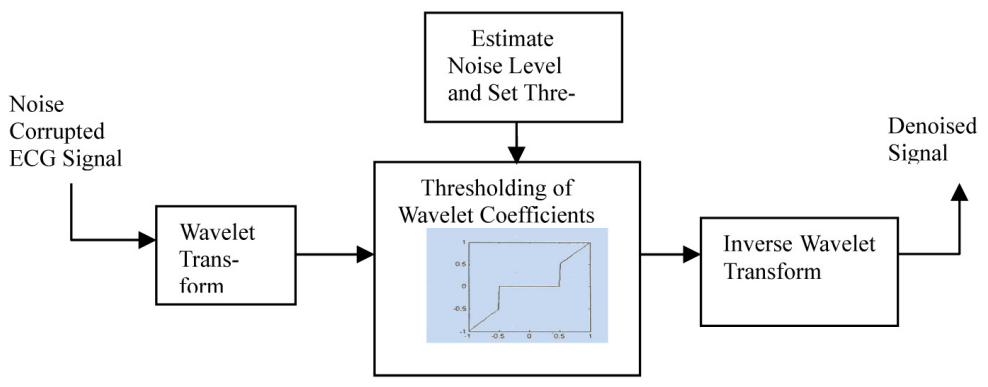

Fig. 1. Wavelet denoising scheme 
to wavelet coefficients. Finally, the signal is reconstructed from these newly adjusted wavelet coefficients. Fig. 1 demonstrates the basic steps of wavelet based denoising.

The four main issues in the wavelet de-noising process are: (1) choice of wavelet basis. (2) choice of depth of decomposition, (3) determination of thresholds at each subband, and (4) choice of suitable thresholding rules. The approach for choosing the above factors is mentioned in the denoising procedure.

\subsection{Kernel Density Estimation}

Density estimation is the construction of an estimate of the density function from the observed data. Density estimation is done by the parametric or nonparametric method. Kernel density estimation is a nonparametric density fitting method. It does not make any assumption about the underlying density. Usually density estimation is done by the well-known histogram method (also known as, block estimation) to find the distribution of a set of values. The two main drawbacks of this method are that it is not smooth and its shape varies with changes in bin widths and end limits. Wavelet coefficients of ECG data do not have a fixed pattern, so nonparametric kernel density estimation is a better option for modeling [16].

The Kernel density estimation method is an important tool in nonparametric density and distribution function fitting. Suppose a dataset containing $X_{1}, X_{2}, \ldots X_{n}$ samples belongs to the probability density of $f(x)$. Then the density estimator of $f(x)$ with kernel $k$ is defined by equation (1) [16-17].

$$
\hat{f}(x)=\frac{1}{n h} \sum_{i=1}^{n} k\left(\frac{x-X_{i}}{h}\right)
$$

Here, $\hat{f}(x)$ is the estimated density, $h$ is the bandwidth (equivalent to the bin width of the histogram), $n$ denotes the number of samples, and $k$ is a bounded nonnegative kernel function satisfying: $\int k(x) d x=1$.

When $k(x)$ is a Gaussian density function then equation (1) becomes a Gaussian kernel density estimation:

$$
k(x)=\frac{1}{\sigma \sqrt{2 \pi}} \exp \left[-\frac{1}{2}\left(\frac{x-\mu}{\sigma}\right)^{2}\right]
$$

where $\mu$ and $\sigma$ defines the mean and the standard deviation of data values respectively.

The selection of optimal bandwidth is critical in density estimation. A large bandwidth gives a smooth estimation (large bias) whereas a small bandwidth gives a spiky one (large variance). The optimal bandwidth is one that minimizes the integral of the square error (MISE) between the estimated density and the true density.

$$
\operatorname{MISE}(f)=E\left[\int(f(x)-f(x))^{2} d x\right]
$$

For the assumption that the true distribution is a Gaussian density and a Gaussian kernel is used, it can be shown that the optimal value of the bandwidth (equation (4)) becomes [17-18] 


$$
h=1.06 \sigma n^{(-1 / 5)}=\frac{\operatorname{median}(|(X-\operatorname{median}(X))|)}{0.6745}\left(\frac{4 n}{3}\right)^{0.2}
$$

where, $h$ is the bandwidth, $n$ represents the number of samples, and $\sigma$ is the sample standard deviation.

\subsection{Denoising Procedure}

The denoising procedure consists of following steps (shown in Fig. 2):

Step 1: Decompose the signal

To decompose the ECG excerpt using discrete wavelet transform, the choice of wavelet basis and the selection of level of decomposition are important. The wavelet domain choice of wavelet function depends on the application and the shape of a signal. Several wavelet families, like Haar, Daudechies, Coiflets, and Symlets, with different scaling functions exist [13-15]. The selection procedure for the choice of an optimal wavelet basis is:

- Decompose each ECG signal using a discrete wavelet transform up to the fourth level using wavelet filters bior4.4, bior5.5, bior6.8, sym8, and db4 (suggested as the best for ECG signal analysis by Garri Clifford).

- Set $\mathrm{cD} 1$ and $\mathrm{cD} 2$ detail coefficients (beyond the ECG bandwidth) to zero.

- Reconstruct the signal from approximations and new detail coefficients. Find the Mean Square Error (MSE) between the original signal and the reconstructed signal.

After an evaluation of the MSE on several noise-corrupted records from the Glasgow database (lead II signal with sampling rate $500 \mathrm{~Hz}$ ), the Daubechies wavelet of order 4 was selected on the basis of minimum MSE (shown in Fig. 3).

The selection of the decomposition level depends on the sampling frequency and the spectral

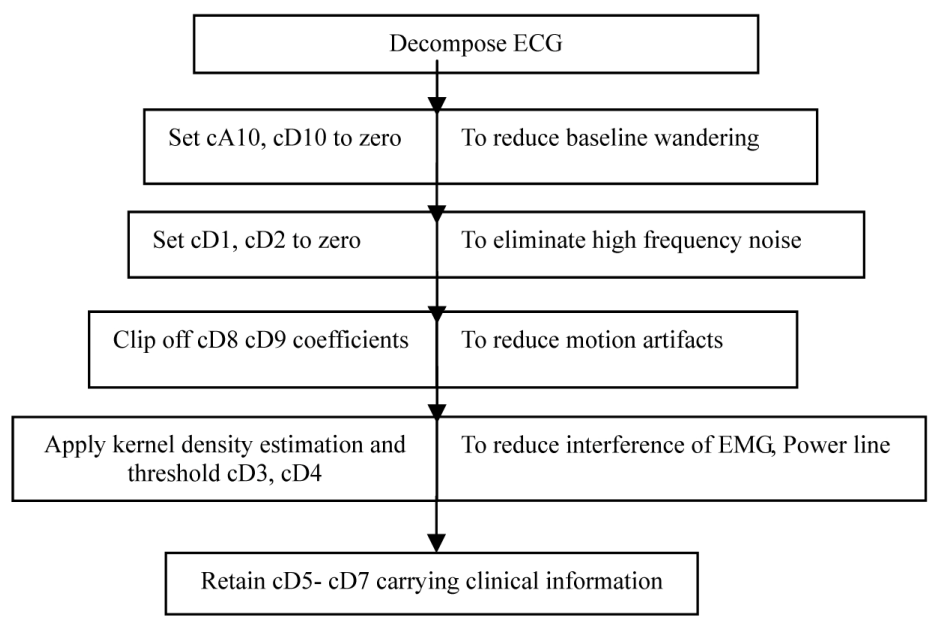

Fig. 2. Steps for implementing the denoising process 


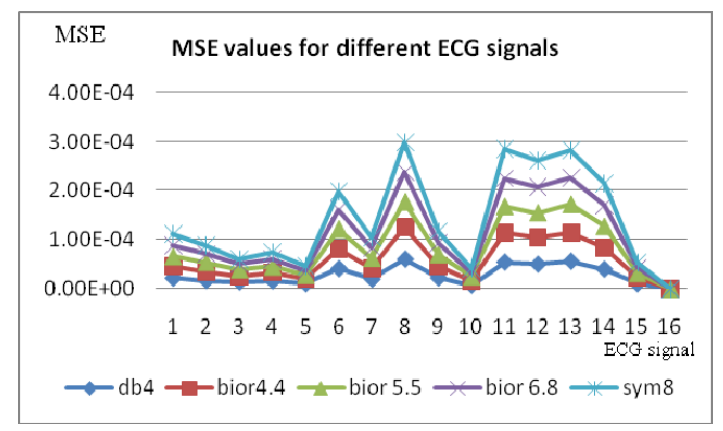

Fig. 3. Mean Square Error calculation for the selection of a wavelet basis

content of the signal to be processed. The banwidth of diagnostic ECG is usually considered as $0.5-100 \mathrm{~Hz}$. Following are the specifications of the noises and artifacts:

- Power line interference (50\% of QRS amplitude, $50 \mathrm{~Hz}$ or $60 \mathrm{~Hz}$ ).

- Electrode contact noise (Maximum recorder output, duration 1s).

- Motion artifacts $(2-5 \mathrm{~Hz}, 500 \%$ of peak).

- Muscle contraction or electromyographic noise (EMG) $(10 \%, 20-10 \mathrm{KHz}$, duration 50ms).

Baseline drift and ECG amplitude modulation with respiration $(0.15-0.3 \mathrm{~Hz}, 15 \%$ of the peak).

- Electrosurgical noise (200\%, $100 \mathrm{KHz}-1 \mathrm{MHz}$, duration 1-10s).

- Instrumentation noise (No ECG output).

To separate out coefficients related to very low frequency noises (baseline wanders), it is practical to select the level of decomposition at ten for the sampling frequencies of $360 \mathrm{~Hz}$ and $500 \mathrm{~Hz}$ (refer Table 1). Table 1 displays the bandwidths of detail and approximation coefficients for the sampling frequencies of 360 and $500 \mathrm{~Hz}$.

Step 2: Correction of baseline wandering

Set the approximation coefficients $\mathrm{cA} 10, \mathrm{cD} 10$ to zero to reduce baseline wandering and ECG amplitude modulation with respiration. Fig. 4 shows the output of this step.

Table 1. Bandwidths of detail coefficients for different sampling frequencies (Fs)

\begin{tabular}{c|c|c}
\hline Coefficients & Fs $=360 \mathrm{~Hz}$ & Fs $=500 \mathrm{~Hz}$ \\
\hline $\mathrm{cD} 1$ & $180-360$ & $250-500$ \\
\hline $\mathrm{cD} 2$ & $90-180$ & $125-250$ \\
\hline $\mathrm{cD} 3$ & $45-90$ & $62.5-125$ \\
\hline $\mathrm{cD} 4$ & $22.5-45$ & $31.25-62.5$ \\
\hline $\mathrm{cD} 5$ & $11.25-22.5$ & $15.62-31.25$ \\
\hline $\mathrm{cD} 6$ & $5.6-11.25$ & $7.8-15.6$ \\
\hline $\mathrm{cD} 7$ & $2.8-5.6$ & $3.9-7.8$ \\
\hline $\mathrm{cD} 8$ & $1.4-2.8$ & $1.95-3.9$ \\
\hline $\mathrm{cD} 9$ & $0.7-1.4$ & $0.97-1.95$ \\
\hline $\mathrm{cD} 10$ & $0.35-0.7$ & $0.48-0.97$ \\
\hline $\mathrm{cA} 10$ & $0.17-0.35$ & $0.24-0.48$ \\
\hline
\end{tabular}



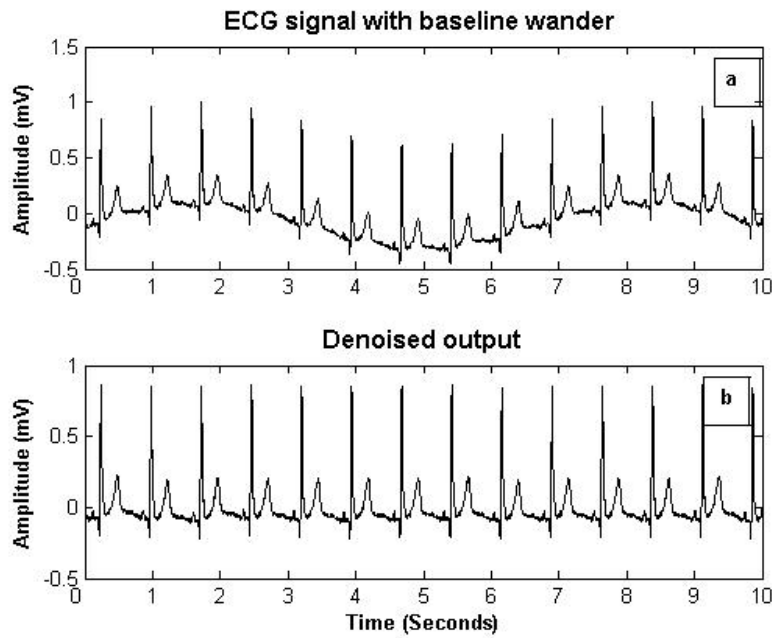

Fig. 4. (a) ECG signal added with baseline wander (b) Denoised output

Step 3: Removal of high frequency

To remove high frequency noise that is present at the first and second level, set the detail coefficients $\mathrm{cD}_{1}$ and $\mathrm{cD}_{2}$ (above ECG bandwidth) to zero. Fig. 5 shows the removal of high frequency noise on ECG signal NOISY_28957.01 (with 500 samples/sec, 12 bit resolution) from the Glasgow database.

Step 4: Reduction of motion artifacts

Motion artifacts have a $2-5 \mathrm{~Hz}$ bandwidth and a magnitude of $500 \%$ of peak. Verify the pres-
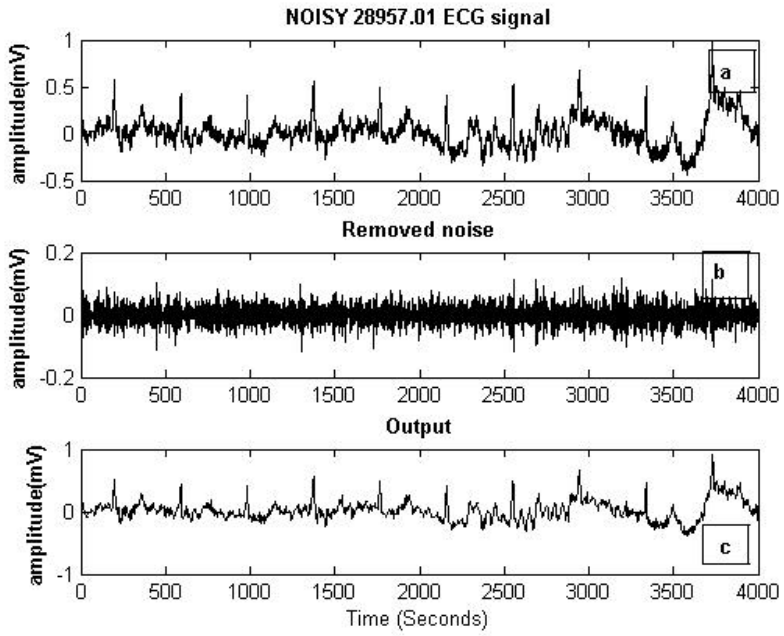

Fig. 5. (a) NOISY_28957.01 ECG sgnal (b) Removed noise by setting cD1 and cD2 to zero (c) Output signal 
ence of motion artifacts by calculating energy in sub-bands cD8, cD9. Remove the very large magnitude cD8, cD9 coefficients above threshold. Fig. 9 illustrates this effect.

Step 5: Reduction of EMG noise

EMG noise contributes small magnitude coefficients in $\mathrm{cD} 3$ and $\mathrm{cD} 4$. To observe the distribution of EMG noise that is present in $\mathrm{cD} 3$ and $\mathrm{cD} 4$, these coefficients need to be modeled using the Kernel density estimation method and the thresholds needs to be determined. For the density estimation of detail coefficients:

- Calculate bandwidth "h" using equation (4).

- Find the minimum and maximum of coefficients.

- Divide the range into $100(N)$ equally spaced points $(x)$.

- Estimate the density for these points using equation (1).

- Obtain the cumulative density function from the estimated density.

- Design thresholds.

Threshold estimation proves the capabilities of wavelet transform for signal denoising. Thresholds are designed separately for Level 3 and 4. Fig. 6 shows the Gaussian kernel density estimation of third level detail coefficients of record NOISY_29000.01 and corresponding normalised cumulative density function of CDF.

It is obvious from Fig. 6 that:

- The peak of distribution, near or at zero, indicates the presence of a small magnitude of detail coefficients with a large frequency (high probability). These coefficients, not defining the morphology of an ECG signal, are easily removed.

- Significant coefficients appearing with less frequency are retained.

- The uprising portion of normalized cumulative distribution function (CDF) belongs to a higher probability and the rest denotes lower probability density values.
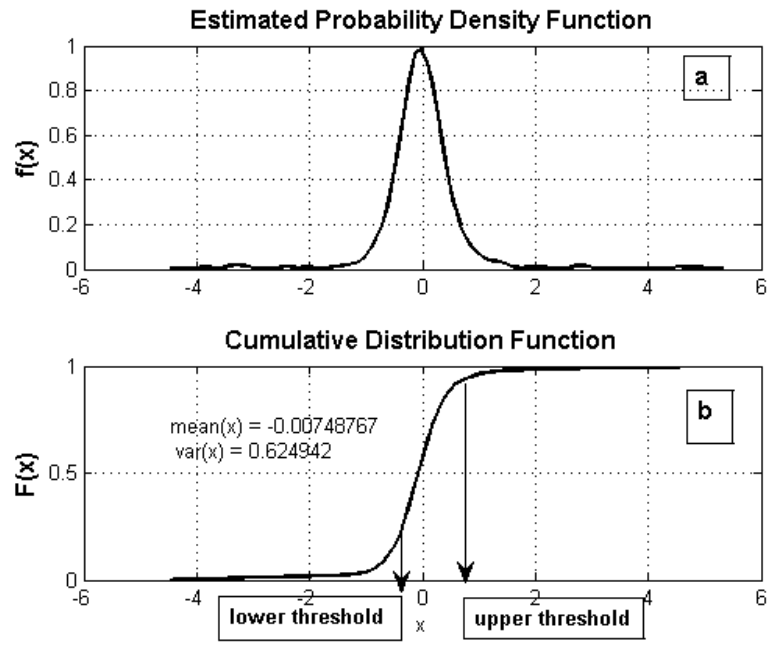

Fig. 6. (a) Gaussian kernel density estimation of CD3 coefficients of NOISY_29000.01 (b) Thresholds obtained on cumulative density function of cD3 by placing $20 \%-80 \%$ window. 
- Therefore, a sliding window is designed over the CDF. The end points of the window result in the lower and upper thresholds on the coefficients. Signal values greater than the lower threshold and less than the upper threshold are removed by hard thresholding. Thus good denoising, as well as compression, is achieved. Fig. 6 shows lower threshold and upper threshold obtained by placing a window width of $20 \%-80 \%$ (finding $x$ co-ordinates for 0.2 and 0.8 ).

Step 6: Retain diagnostic information

Basically ECG wave components, the QRS complex, the P wave, and the T wave, reside cD5 onwards. Therefore preserve the coefficients cD5-cD7carrying clinical information.

\section{RESULTS AND Discussion}

The proposed algorithm basically reduces low and high frequency noise that is present in ECG records. The method was evaluated on highly corrupted MIT-BIH arrhythmia database records and noise corrupted records from the Glasgow database. Noise free records from the Glasgow database were used for validating the performance of our method on the basis of diagnostic information. First level and second level detail coefficients along with approximation coefficients were set to zero, as these coefficients do not carry ECG information. Third and fourth level coefficients were modeled using the kernel density estimation.

For modeling sub-band coefficients, the three kernels that attempted were: Exponential, Cauchy, and Gaussian. Gaussian kernel density with bounded support was proved to be a better model among the three kernels on the basis of goodness of fit. The $\mathrm{p}$ value was found to be high $(>>0.05)$ by the Kolmogorov-Smirnov test. So the null hypothesis, which is the distribution of the wavelet sub-band coefficients, was equal to the density estimation using the Gaussian kernel and was accepted.

For the Glasgow database records, thresholds of third and fourth sub-bands were obtained by placing window widths $20 \%-80 \%$ and $30 \%-70 \%$ on normalised CDFs respectively. Lower and upper thresholds calculated from the noisy ECG records from the Glasgow database are shown in Table 2. The window widths were finalised after numerous experiments while keeping an eye over PRD (below $8 \%$ ) and diagnostic distortion (below 0.1).

Table 2. Assessment of thresholds obtained for different window widths in ECG records

\begin{tabular}{l|c|c|c|c|c|c}
\hline \multirow{2}{*}{$\begin{array}{c}\text { Single lead rec- } \\
\text { ord. }\end{array}$} & \multicolumn{2}{|c|}{$\begin{array}{c}\text { cD3 -thresholds with } \\
20 \%-80 \% \text { window. }\end{array}$} & \multirow{2}{*}{$\begin{array}{c}\text { cD3 Third level } \\
\text { magnitude range. }\end{array}$} & \multicolumn{2}{c|}{$\begin{array}{c}\text { cD4- thresholds with } \\
30 \%-70 \% \text { window. }\end{array}$} & \multirow{2}{*}{$\begin{array}{c}\text { cD4 Fourth level } \\
\text { magnitude range. }\end{array}$} \\
\cline { 2 - 3 } & Lower & Upper & & Lower & Upper & \\
\hline NOISY_29000.01 & -0.37 & 0.31 & {$[-4.2,4.9]$} & -0.23 & 0.27 & {$[-6.56,9.33]$} \\
\hline NOISY_33010.01 & -0.27 & 0.27 & {$[-2.68,1.78]$} & -0.44 & 0.46 & {$[-5.67,5.69]$} \\
\hline NOISY_31264.01 & -0.65 & 0.80 & {$[-7.2,4.47]$} & -0.79 & 0.65 & {$[-9.08,12.08]$} \\
\hline NOISY_28957.01 & -0.47 & 0.45 & {$[-2.50,2.43]$} & -0.58 & 0.45 & {$[-5.85,5.33]$} \\
\hline NOISY_31103.01 & -0.62 & 0.64 & {$[-5.10,3.37]$} & -0.46 & 0.61 & {$[-13.35,11.08]$} \\
\hline NOISY_31228.01 & -0.36 & 0.42 & {$[-4.07,1.5]$} & -0.40 & 0.33 & {$[-7.18,6.23]$} \\
\hline NOISY_31361.01 & -0.16 & 0.15 & {$[-4.7,2.8]$} & -0.31 & 0.13 & {$[-10.42,10.91]$} \\
\hline
\end{tabular}



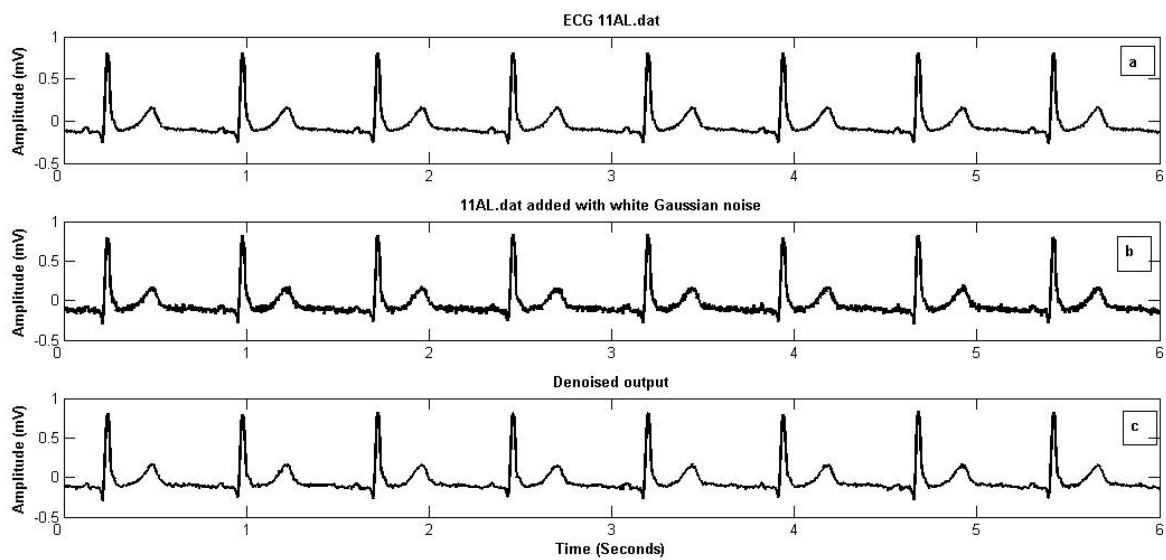

Fig. 7. (a) ECG signal 11AL.dat, (b) Signal added with white Gaussian noise, (c) Denoised signal

White Gaussian noise (usually used to model Electromyographic noise), random noise, and mains noise were added for testing the algorithm. The result of our method on white Gaussian noise corrupted 11AL.dat of the Glasgow database and is shown in Fig. 7.

Wavelet coefficients due to mains noise $(50 \mathrm{~Hz})$ belong to $\mathrm{cD} 3$ or $\mathrm{cD} 4$ for sampling frequencies $360 \mathrm{~Hz}$ (MIT-BIH database) or $500 \mathrm{~Hz}$ (Glasgow database) respectively. Therefore the thresholding of $\mathrm{cD} 3$ and $\mathrm{cD} 4$ coefficients have provided the efficient removal of mains noise. Fig. 8 shows waveforms of noise free ECG - 11AL.dat from the Glasgow database, mains noise corrupted ECG, and denoised output.

The proposed method worked satisfactorily on records from the Glasgow database. Fig. 9 illustrates the denoised output of our scheme on NOISY_31807.01.

The quality metrics of denoising methods, SNR (Signal to Noise Ratio), and PRD (Percentage
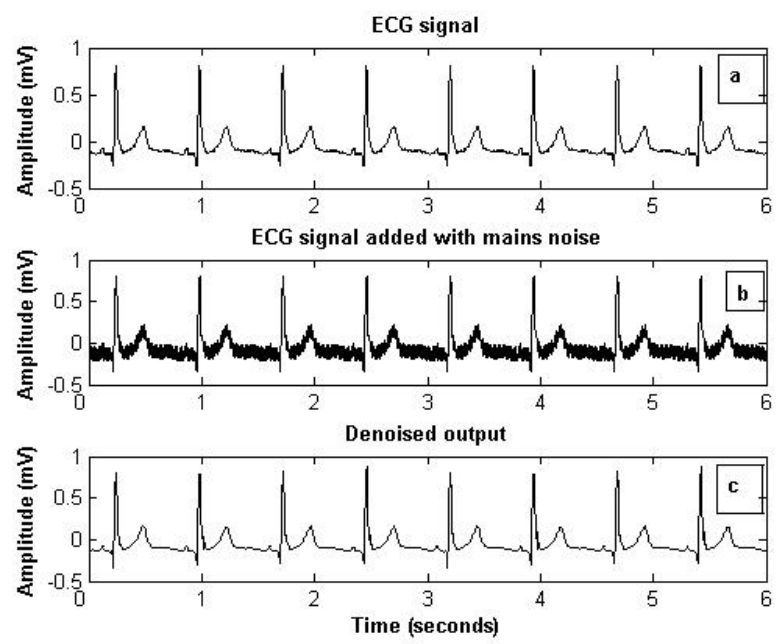

Fig. 8. (a) Original 11AL.dat, (b) Signal added with mains noise, (c) Denoised 11AL.dat 

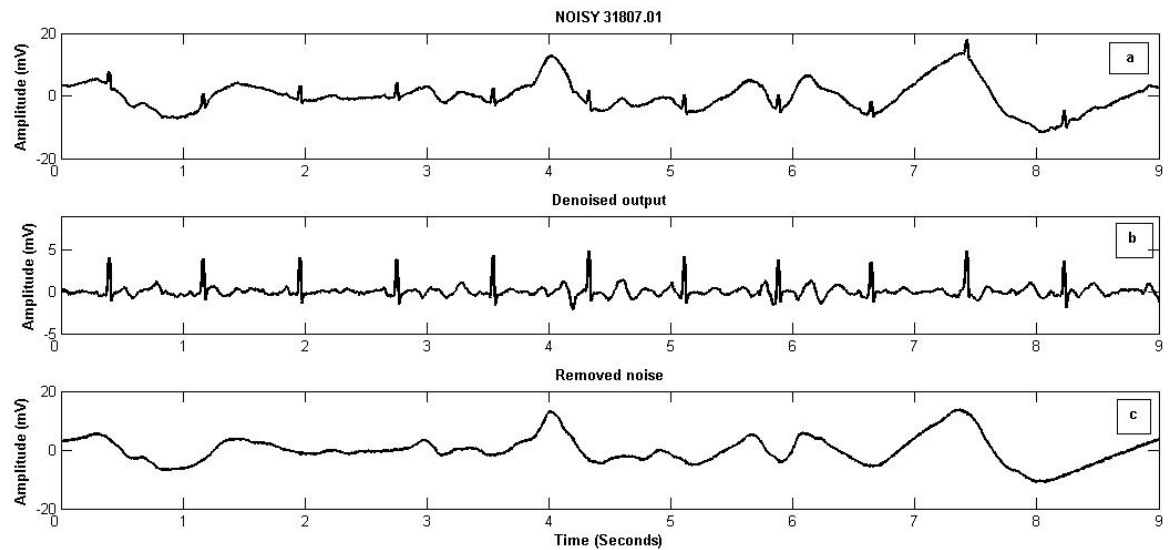

Fig. 9. (a) Noise corrupted 31807.01 signal, (b) Denoised signal, (c) Removed noise

RMS difference) are defined by (5) and (6) respectively.

$$
\begin{gathered}
S N R=10 \log \frac{\sum_{n=1}^{N} \tilde{x}(n)^{2}}{\sum_{n=1}^{N}(x(n)-\tilde{x}(n))^{2}} \\
P R D=100 \times \sqrt{\sum_{n=1}^{N}[x(n)-\tilde{x}(n)]^{2} / \sum_{n=1}^{N}[x(n)]^{2}}
\end{gathered}
$$

where $x(n)=$ original ECG signal, $\tilde{x}(n)=$ reconstructed ECG signal after denoising

The method developed by Mikhled Alfaouri and Khaled Daqrouq was evaluated on ECG records by adding random noise and was tested for the two quality metrics of SNR and PRD [5]. To compare our method with Alfouri's method, 25 ECG records (each record had random noise added to it) from the Glasgow database were used. The SNR and PRD values of our method were found to be better, as illustrated in Fig. 10 and Fig. 11, respectively. The PRD values were higher by using Alfouri's method, as detail coefficients above thresholds were set to value one at all levels.

The performance of the proposed denoising algorithm was endorsed by evaluating diagnostic distortion after employing our scheme. The clinical information of ECG signals is mostly present in P-waves, QRS complexes, and T-waves. The parameters describing these waves are called "features." The features were obtained by first detecting fiducial points (P, Q, R, S, and T) based on the selective coefficient method [14]. Fig. 12 shows the detection of fiducial points for the 11AL.dat ECG record from the Glasgow database.

Features were calculated from magnitudes and positions of fiducial points. Table 3 displays ten essential features for cardiac arrhythmia detection, which were used for defining feature vector F [19]. 


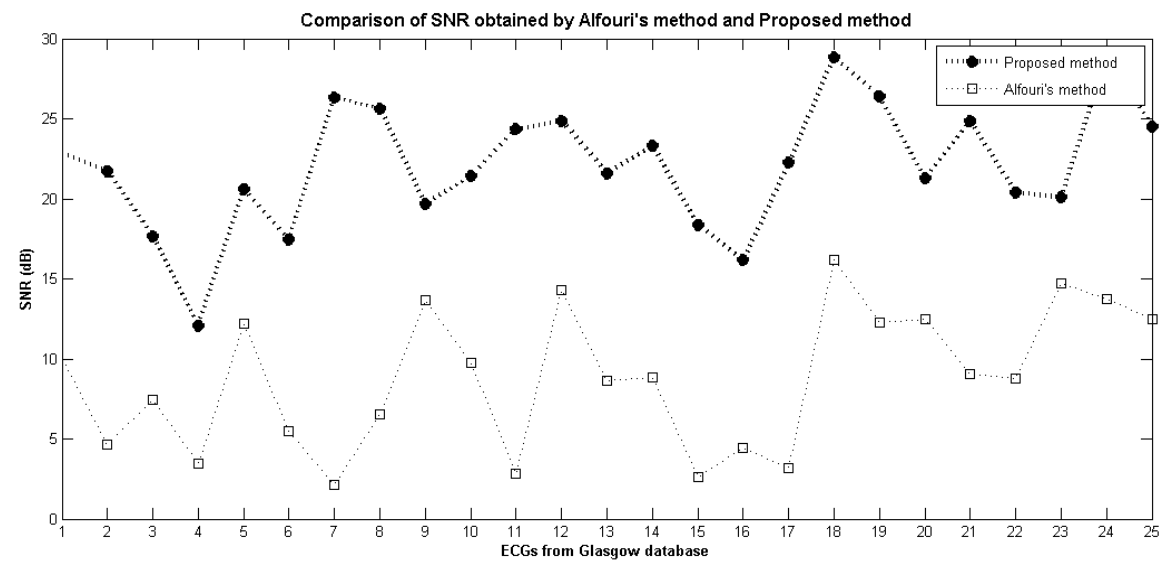

Fig. 10. Comparison of SNRs evaluated by the ECG records from the Glasgow database

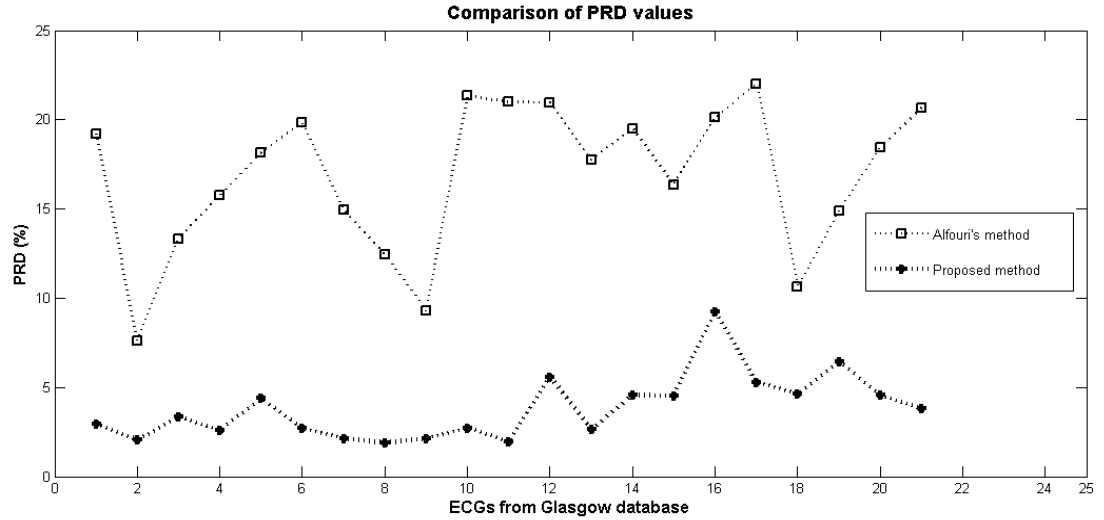

Fig. 11. Comparison of PRD values evaluated by the ECGs from the Glasgow database
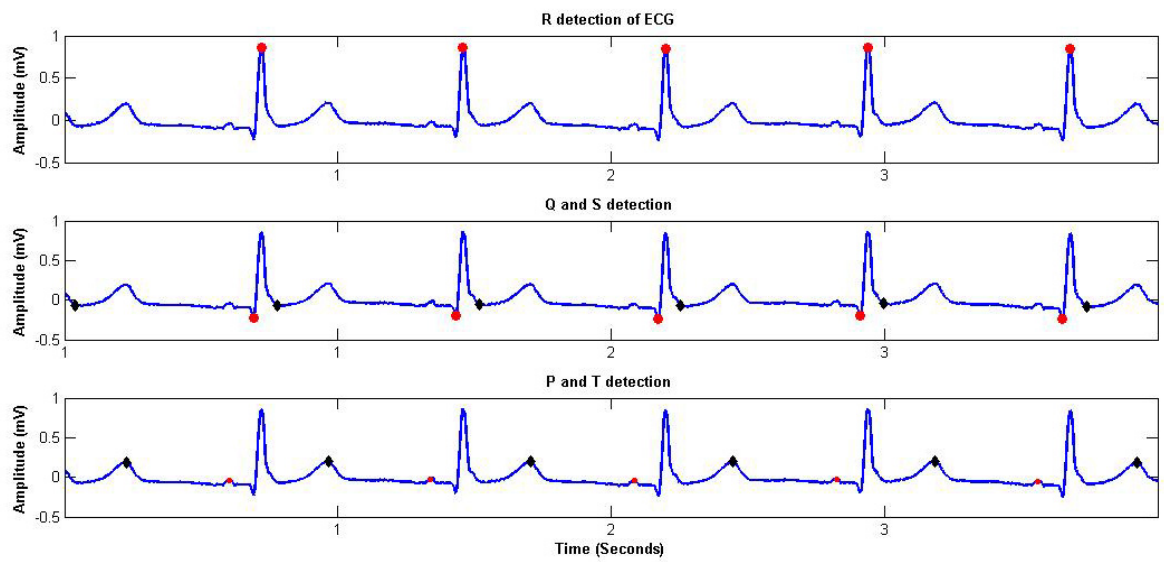

Fig. 12. (a) R detection of $11 \mathrm{AL}$.dat, (b) $\mathrm{Q}$ and $\mathrm{S}$ detection, (c) $\mathrm{P}$ and $\mathrm{T}$ detection 
Table 3. Features evaluated in ECG records

\begin{tabular}{c|c|l}
\hline Serial No. & Feature's Notation & \multicolumn{1}{c}{ Feature's Description } \\
\hline 1 & Height_QR & The amplitude between Q and R in a QRS complex \\
\hline 2 & Height_RS & The amplitude between R and S in a QRS complex \\
\hline 4 & QRS_duration, & The time duration between Q and S in a QRS complex \\
\hline 5 & Ratio_RR & $\begin{array}{l}\text { Ratio of } \mathrm{RR}_{\mathrm{s}} \text { and } \mathrm{RR}_{\mathrm{a}}, \mathrm{RR}_{\mathrm{s}} \text { is the length of RR interval } \\
\text { and } \mathrm{RR}_{\mathrm{a}} \text { is the average length of all RR intervals }\end{array}$ \\
\hline 6 & Slope_QR & The slope between Q and R in a QRS complex \\
\hline 7 & Slope_RS & The slope between $\mathrm{R}$ and S in a QRS complex \\
\hline 8 & P_amplitude & The amplitude of the P wave \\
\hline 9 & PR_duration & The time duration between P and R \\
\hline 10 & T_amplitude & The amplitude of the T wave \\
\hline
\end{tabular}

To observe the effect of denoising on features, the procedure listed below was implemented. Its steps consist of:

- Extract feature vector $\mathrm{F}_{\mathrm{o}}$ having 10 features from each noise free ECG.

- Add random noise to the ECG signal and apply proposed denoising scheme.

- Extract feature vector $F_{d}$ from the denoised ECG and calculate the MSE using equation (7).

The MSE (mean square error) between the feature vectors of the original and denoised signal is related to the diagnostic distortion and is given by:

$$
M S E=\frac{1}{N} \sum_{k=1}^{N}\left(F_{d}(k)-F_{o}(k)\right)^{2}
$$

$N=$ number of features, $F_{\mathrm{o}}=$ Feature vector of original, $F_{\mathrm{d}}=$ Feature vector of the denoised signal.

Fig. 13 displays the MSE values obtained from 30 records by the above procedure. It was observed that most of MSE values were below 0.05 and indicated negligible variation in their features.

In the wavelet based denoising process, noise coefficients are set to zero, which leads to good compression. This parameter is named the Percentage of Zeros (PZ) and is described by equation $(8)$.

$$
P Z=\frac{\text { Total detail coefficients replaced by zero } \times 100}{\text { Total number of detail coefficients }}
$$

Table 4 demonstrates SNR and PZ values for ambulatory records when added with random noise. The highly corrupted seven ambulatory ECG signals: 101, 103, 106, 116, 123, 202, 232 were selected from the MIT-BIH Arrhythmia Database [20]. Fig. 14 depicts comparison of PZ values with other method calculated over 45 records from Glasgow database. 


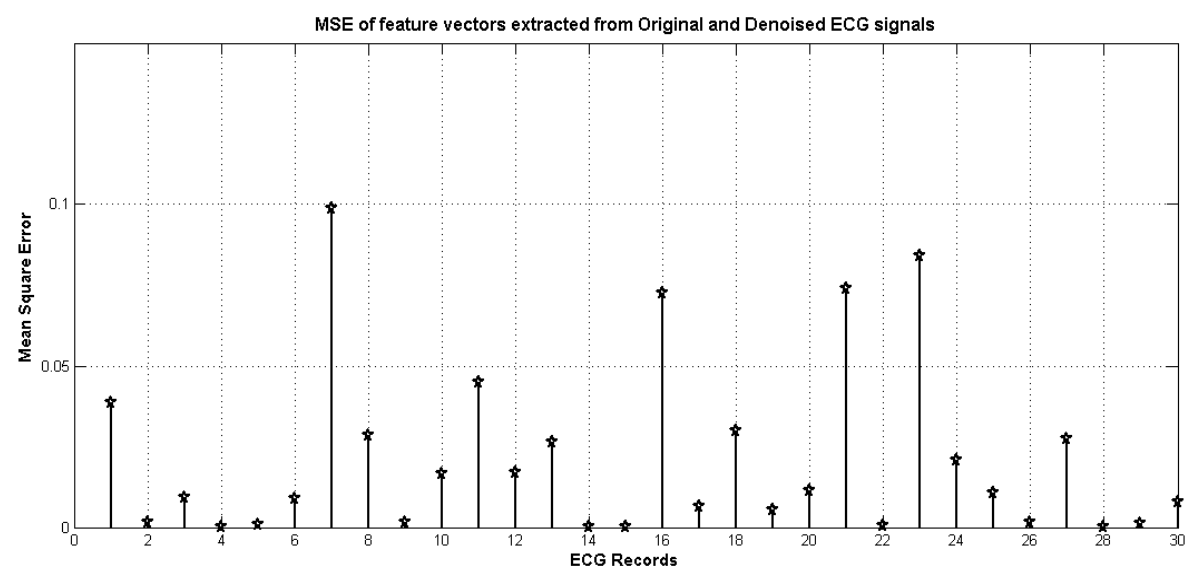

Fig. 13. MSE values obtained from 30 Glasgow databases

Table 4. Performance measures evaluated in the Ambulatory ECG records

\begin{tabular}{c|c|c}
\hline \multirow{2}{*}{ Ambulatory Records } & \multicolumn{2}{|c}{ Proposed Method } \\
\cline { 2 - 3 } & SNR (dB) & PZ (\%) \\
\hline 101.dat & 43.268 & 83.927 \\
\hline 103.dat & 42.715 & 81.997 \\
\hline 106.dat & 40.633 & 85.339 \\
\hline 116.dat & 38.718 & 84.245 \\
\hline 123.dat & 42.167 & 84.782 \\
\hline 202.dat & 45.917 & 84.703 \\
\hline 232.dat & 44.636 & 84.882 \\
\hline
\end{tabular}

\section{CONCLUSION}

Basically an orthogonal wavelet transform compresses the "energy" of the signal in a relatively small number of large coefficients. However, the energy of noise is dispersed throughout the transform, giving a really large number of small coefficients. Therefore, a new and robust thresholding strategy based on the modeling of sub-band coefficients is proposed in this study. The method is found to be the most suitable for ECG, as EMG noise is prevalent in the lower four levels. After the removal of baseline wander and motion artifacts, the approach of modeling coefficients to design thresholds was found to be proper from experimentation.

Testing on several records proved that our method of designing thresholds has better results, as compared to other methods. This method has proposed an appropriate estimation of a lower threshold (a small negative value) and an upper threshold (a small positive value) in third and fourth sub-bands, which causes the removal of noise values within range. The method reduces noise effectively and reliably while preserving important features at the same time. The study considered all types of noise and artifacts and has provided improved SNR and PRD, along with 
considerable PZ. The adaptability towards different sampling frequency can be achieved by changing the sub-bands of treatment and window widths for specifying thresholds. Instead of hard thresholding for shrinkage, other nonlinear shrinkage functions can be used. The combination of density functions can model the heavy-tailed nature of wavelet coefficients in a better way.

\section{REFERENCES}

[1] Gary M. Friesen, Thomas C. Jannett, Manal Afify Jadallah, Stanford L. Yates, Stephen R. Quint, H. Troy Nagle, "A Comparison of the Noise Sensitivity of Nine QRS Detection Algorithms", IEEE Transactions On Biomedical Engineering, 1990, Vol.37, pp.85-98.

[2] Brij N. Singh, Arvind K. Tiwari, "Optimal selection of wavelet basis function applied to ECG signal denoising”, Digital Signal Processing,2006, Vol.16, pp.275-287.

[3] Huseyin Tirtom, Mehmet Engin, Erkan Zeki Engin, "Enhancement of time-frequency properties of ECG for detecting micropotentials by wavelet transform based method"; Expert Systems with Applications, 2008, Vol.34, pp.746-753.

[4] Donoho D. L., "De-Noising by soft-thresholding”, IEEE Transactions on Information Theory, 1995, Vol.41, pp.613-627.

[5] Mikhled Alfouri,Khaled Daqrouq, "ECG Signal denoising by Wavelet Transform Thresholding”, American Journal of Applied Sciences, 2008. 5, pp.276-281.

[6] Daniel Novak,David Cuesta Frau, Vladimir Eck, Juan C. Perez-Cortes, Gabriela, Andreu-García, "Denoising electrocardiogram signal using adaptive wavelets", Digital Signal Processing, 2006, Vol.16, pp.275-287.

[7] Yunfeng Wu, Rangaraj M. Rangayyan, Yachao Zhouc, Sin-Chun Ngd, "Filtering Electrocardiographic signals using an unbiased and normalized adaptive noise system”, Medical Engineering \& Physics, 2008, Vol.31, pp.17-26.

[8] K.Daqrouq, "ECG Baseline Wandering Reduction using Discrete Wavelet Transfrom", Applied Journal of Information Technology, 2005. 4, pp.989-995.

[9] D.S. Brito, Enio Aguiar, Fausto Lucena, R.C.S.Freire, Yoshifumi Yasuda, A.K.Barro, "Influence of low frequency noise in adaptive estimation using the LMS algorithm", Signal Processing, 2009, pp.933-940.

[10] M. Kania,M. Fereniec,R. Maniewski, “Wavelet Denoising for Multi-lead High Resolution ECG Signals”, Measurement Science Review, 2007, Vol.7, Section 2, No.4, pp.30-33.

[11] L.N. Sharma, S. Dandapat, A. Mahanta, "ECG signal denoising using higher order statistics in Wavelesubbands”, Biomedical Signal Processing and Control, 2010, Vol.5, pp.214-222.

[12] Muhammad ZiaUrRahman, RafiAhamedShaik, D.V.RamaKotiReddy, "Efficient signbasednormalizedadaptive filtering techniques for cancelation of artifacts in ECG signals:Application to wireless biotelemetry”, Signal Processing, 2011. 9, pp.225-239.

[13] Andrew T.Reisner, Gari Clifford [online]: http://www.robots.ox.ac.uk/ gari/code.html

[14] S. Z. Mahmoodabadi, A. Ahmadian1, M. D. Abolhasani, "ECG Feature Extraction using Daubechies Wavelets", Proceedings of fifth IASTED International conference, Visualization, Imaging, Image Processing, 2005, pp.343-348.

[15] K.I.Ramchandran, K.P.Soman, 'Insight into wavelets from theory to practice', Prentice Hall Ltd, Second Edition, 2006.

[16] Earl Gose, Richard Johnsonbaugh, Steve Jost, Pattern Recognition and Image Analysis, Prentice Hall of India, 2005.

[17] Thomas Ledl, "Kernel Density Estimation: Theory and Application in Discriminant Analysis", Austrian journal of statistics, 2004, Vol.33, pp.267-279.

[18] B.W. Silverman, "Density Estimation For Statistics And Data Analysis", Monographs on Statistics and Applied Probability, London, Chapman and Hall, 1986, pp.1-22. 
[19] Yun-Chi Yeh, Wen-June Wanga, Che Wun Chiou, "Feature selection algorithm for ECG signals using Range-Overlaps Method", Expert Systems with Applications, 2010, Vol.37, pp.3499-3512.

[20] Yunfeng Wu, Rangaraj M. Rangayyan, and Sin-Chun Ng, "Cancellation of Artifacts in ECG Signals Using a Normalized Adaptive Neural Filter",Proceedings of the 29th Annual International Conference of thes IEEE EMBS, 2007, pp.2552-2555.

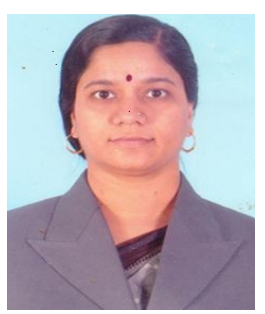

\section{Shubhada Ardhapurkar}

She received the Bachelor's degree (1987) and the Master of Engineering degree (1994) in Electronics from Amaravati University and Marathwada University respectively. She is currently pursuing Ph.D. in ECG Classification using Signal Processing Techniques from S.G.G.S. Institute of Engineering and Technology, Nanded, Maharashtra, India.

She has 21 years of teaching experience and is currently working as Associate Professor in Electronics and Telecommunication Engineering Department at International Centre of Excellence in Engineering and Management at Aurangabad, Maharashtra, India. She has published 10 research papers in Journals and Conferences.Her research interests include Signal processing, VLSI, Communication and Neural networks. She is member of IEEE.

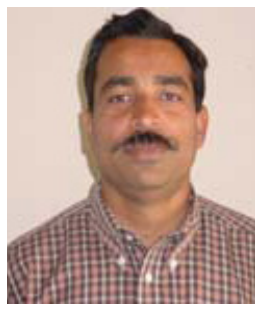

\section{Ramchandra Manthalkar}

He received the Bachelor's and Master of Engineering degree in Electronics from Marathwada University, Aurangabad in 1988 and 1994 respectively. He later received Ph.D. degree from Indian Institute of Technology, Kharagpur in Image Processing Engineering in 2003.

He has 24 years of teaching experience and is currently working as Professor and Head in Electronics and Telecommunication Engineering Department at S.G.G.S. Institute of Engineering and Technology, Nanded, Maharashtra, India. He has published more than 30 research papers in peer reviewed Journals and Conferences. He is currently guiding 4 research scholars for their Ph.D. work. His Biography is included in the current edition of Marquis who's who in Science and Engineering and was included in the International Biographical Centre of Cambridge, England as "Outstanding Scientist of 21st century" His research interests include Biomedical Signal and Image processing, Analog and Mixed signal VLSI Design and Biometrics. 


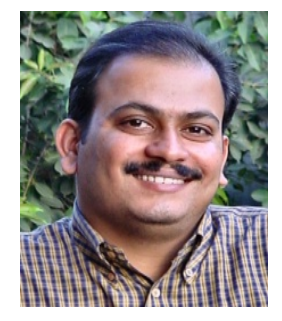

\section{Suhas Gajre}

He received the Bachelor's and Master's of Engineering degree in Electronics from Marathwada University, Aurangabad in 1990 and 1995respectively. He later received Ph.D. degree from Indian Institute of Technology, Delhi in Biomedical Engineering in 2007.

He has 21 years of teaching experience and is currently working as Associate Professor in Electronics and Telecommunication Engineering Department at S.G.G.S. Institute of Engineering and Technology, Nanded, Maharashtra, India. He has published more than 10 research papers in peer reviewed Journals and Conferences. He is currently guiding 4 research scholars for their Ph.D. work. His research interests include Biomedical Signal and Image processing, and Analog and Mixed signal VLSI Design. 